\title{
Delayed Two-Stage Hybrid Management for a Thoracic Aorta Pseudoaneurysm in Polytrauma
}

\author{
Dae Sung Ma, Sung Youl Hyun, Yang Bin Jeon \\ Department of Trauma Surgery, Gachon University Gil Medical Center, Incheon, Korea
}

We successfully managed a traumatic thoracic pseudoaneurysm using a delayed two-stage hybrid approach in polytrauma. (Trauma Image Proced 2016(1):29-31)

Key Words: Aneurysm, False; Endovascular Procedures; Aortic Aneurysm, Thoracic; Wounds and Injuries

\section{CASE}

A 19-year-old man who was on the backseat of a motorcycle involved in an accident was transferred from a regional hospital to our trauma center for management of a thoracic aortic injury. Chest computed tomography (CT) revealed a pseudoaneurysm at the proximal descending thoracic aorta with a mediastinal hematoma. The pseudoaneurysm was located near the origin of the left subclavian artery (Fig. 1.). Abdominoplevic CT revealed concomitant severe hepatic laceration (grade IV) (Fig. 2.). He was admitted to the trauma intensive care unit and managed pursuant to treating the aortic dissection. On the following day, prophylactic embolization at both hepatic arteries was performed because of aggravating abdominal distension. Therefore, we planned a delayed two-stage hybrid approach for the pseudoaneurysm. After day 9, we performed arch debranching with an 8-mm artificial graft $\left(\right.$ Hemashield $\left.^{\mathbb{R}}\right)$ of the left common carotid artery and left subclavian artery (Fig. 3.) in the operating room. Three days after the debranching, thoracic endovascular aortic repair (TEVAR) was performed using 26-110 $\mathrm{mm}$ Seal stent graft (S\&G Biotech, Seongnam, Korea) in the intervention room (Fig. 4.). Dysarthria was observed after the TEVAR. Brain CT angiography and consultation with a neurologist revealed occlusion of the right superior cerebellar artery due to the embolus. Anticoagulation was started and symptoms improved. The patient was discharged without complications on day 25 after admission.

\section{DISCUSSION}

Blunt aortic injury is the second leading cause of death (1). According to management for blunt aortic injury according guidelines (2), it is feasible to delay repair for polytrauma patients with other severe concomitant injuries, especially if they are hemodynamically stable. Since 1997, endovascular repair has been widely used for management of aortic injury, and high success

Received: June 27, 2016 Revised: July 15, 2016 Accepted: August 2, 2016

Correspondence to: Yang Bin Jeon, MD

Department of Trauma Surgery, Gachon University Gil Medical Center, 21, Namdon-daero, 774 Beon-gil, Namdong-gu, Incheon 21565, South Korea

Tel: 82-32-460-3010, Fax: 82-32-460-2372, E-mail: junyb@gilhospital.com

Copyright (c) 2016 Korean Association for Research, Procedures and Education on Trauma. All rights reserved.

(9) This is an open-access article distributed under the terms of the Creative Commons Attribution Non-Commercial License (http://creativecommons.org/ licenses/by-nc/4.0) which permits unrestricted noncommercial use, distribution, and reproduction in any medium, provided the original work is properly cited 

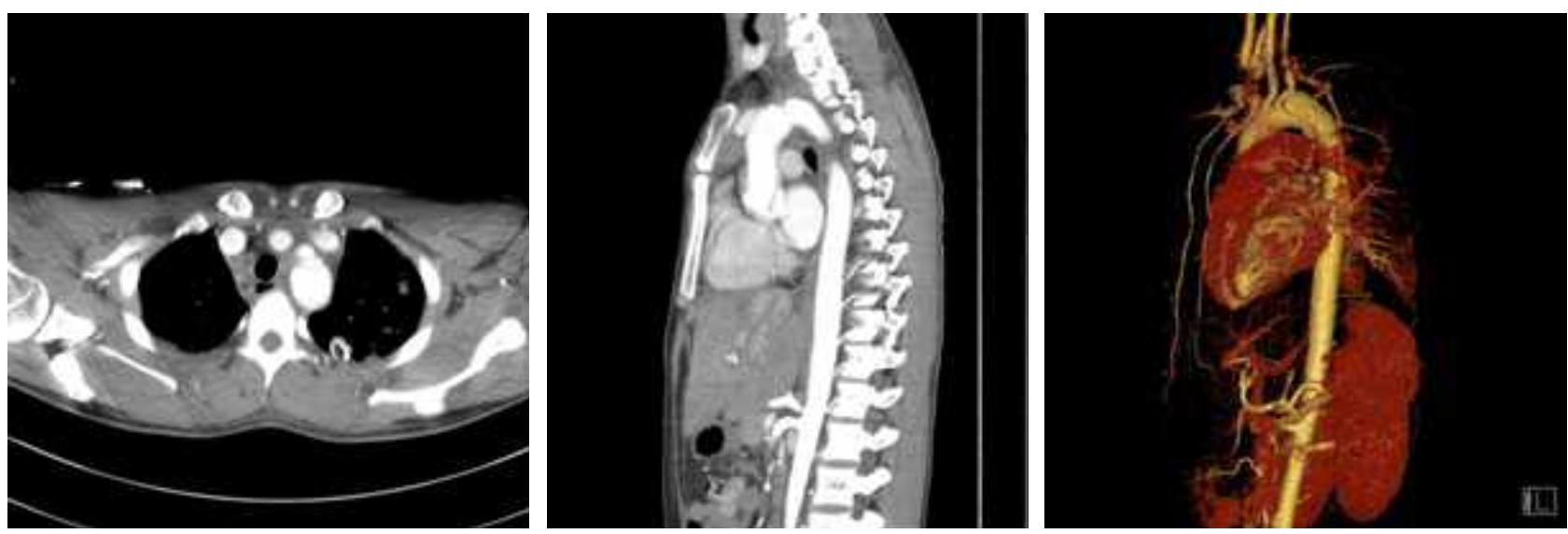

Fig. 1. Traumatic pseudoaneurysm at near left subclavian artery origin

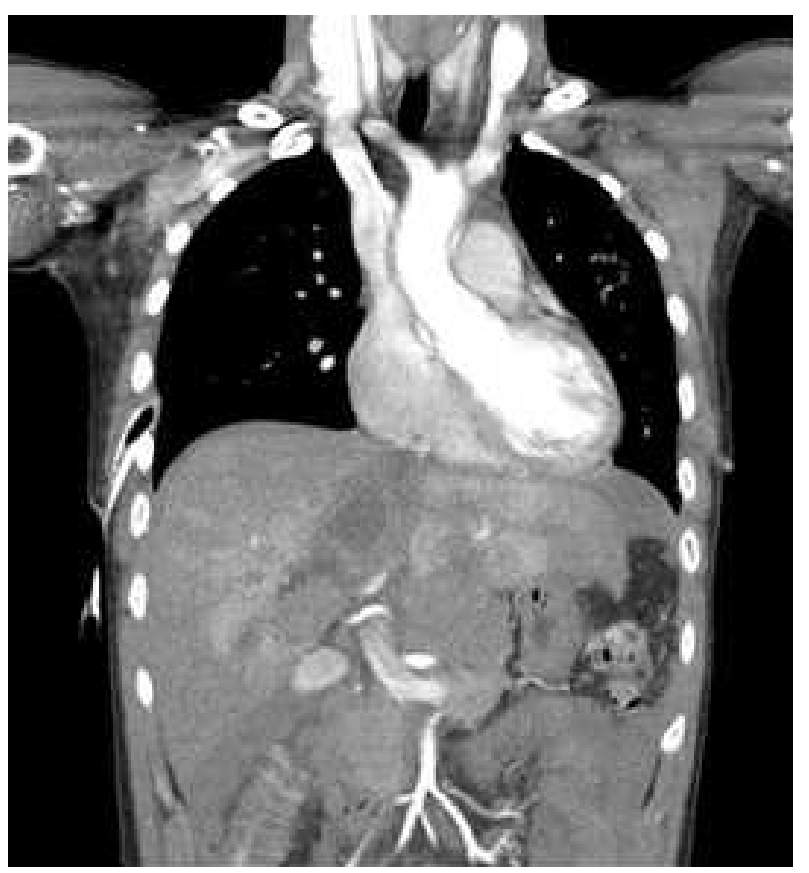

Fig. 2. Abdominoplevic computed tomography revealing hepatic laceration (grade IV)

rates and low mortality rates compared with surgical treatment have been reported (3). However, traumarelated injuries located at an acute angle of the aortic arch and a short distance from arch vessels may predict a higher risk of endograft failure (4). Mosquera et al. (5) reported that $37.9 \%$ of the conservative group had an aortic-related complication in long-term results, and the survival rate was decreased at 10 years $(75.6 \%$ at 1 year, $72.3 \%$ at 5 years, and $66.7 \%$ at 10 years). Survival

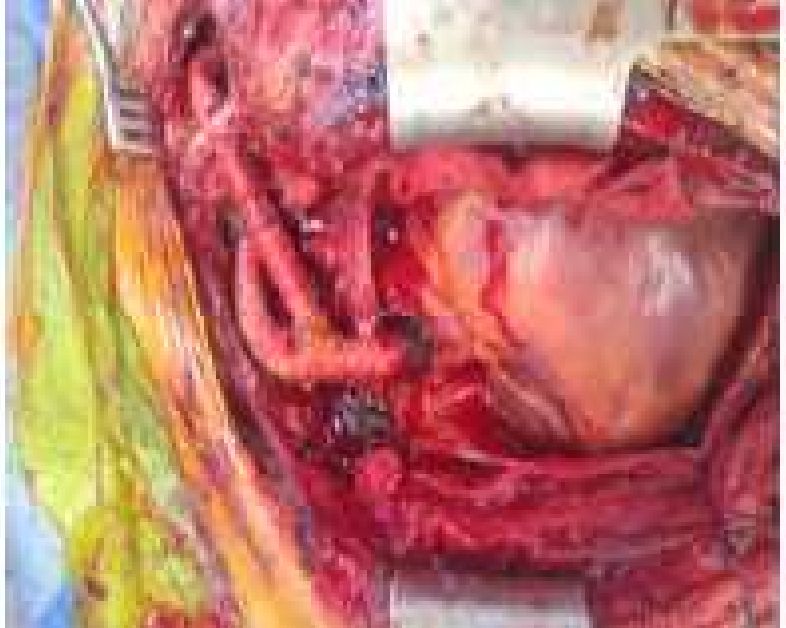

Fig. 3. Debranching the common carotid and superior cerebellar arteries

in the surgical group remained at $77.2 \%$ at 1,5 , and 10 years and in the endovascular group was $85.7 \%$ at 1 and 5 years (5). A hybrid approach for injuries that are unsuitable for endovascular repair alone is applicable for thoracic aortic aneurysm (6). A delayed two-stage hybrid approach for a blunt thoracic aortic injury needing repair may be a feasible strategy for management of polytrauma patients.

\section{CONFLICT OF INTEREST}

No potential conflict of interest relevant to this article was reported. 


\section{REFERENCES}

1. Smith RS, Chang FC. Traumatic rupture of the aorta: still a lethal injury. American journal of surgery. 1986;152(6): 660-3.

2. Fox N, Schwartz D, Salazar JH, Haut ER, Dahm P, Black $\mathrm{JH}$, et al. Evaluation and management of blunt traumatic aortic injury: a practice management guideline from the Eastern Association for the Surgery of Trauma. The journal of trauma and acute care surgery. 2015;78(1):136-46.

3. Xenos ES, Minion DJ, Davenport DL, Hamdallah O, Abedi $\mathrm{NN}$, Sorial EE, et al. Endovascular versus open repair for descending thoracic aortic rupture: institutional experience and meta-analysis. European journal of cardio-thoracic surgery : official journal of the European Association for
Cardio-thoracic Surgery. 2009;35(2):282-6.

4. Neschis DG, Moainie S, Flinn WR, Scalea TM, Bartlett ST, Griffith BP. Endograft repair of traumatic aortic injury-a technique in evolution: a single institution's experience. Annals of surgery. 2009;250(3):377-82.

5. Mosquera VX, Marini M, Lopez-Perez JM, Muniz-Garcia J, Herrera JM, Cao I, et al. Role of conservative management in traumatic aortic injury: comparison of long-term results of conservative, surgical, and endovascular treatment. The Journal of thoracic and cardiovascular surgery. 2011;142(3): 614-21.

6. Zhou W, Reardon M, Peden EK, Lin PH, Lumsden AB. Hybrid approach to complex thoracic aortic aneurysms in high-risk patients: surgical challenges and clinical outcomes. Journal of vascular surgery. 2006;44(4):688-93. 